\title{
Targeting therapy of hepatocellular carcinoma with doxorubicin prodrug PDOX increases anti-metastatic effect and reduces toxicity: a preclinical study
}

Qun Wang ${ }^{1+}$, Yan-Jun Zhong ${ }^{1+}$, Jing-Ping Yuan ${ }^{1+}$, Li-Hua Shao $^{1}$, Jue Zhang ${ }^{1}$, Li Tang $^{1}$, Shao-Ping Liu', Ya-Ping Hong ${ }^{2}$, Raymond A Firestone ${ }^{3}$ and Yan Li ${ }^{1^{*}}$

\begin{abstract}
Background: This study was to investigate the effects and safety of cathepsin B-cleavable doxorubicin (DOX)-prodrug (PDOX) for targeting therapy of metastatic human hepatocellular carcinoma (HCC) using DOX as a positive control drug.

Methods: The orthotopic nude mice model of highly metastatic HCC was established and the animals were randomized and treated with PDOX, DOX and saline, respectively. Hematology, biochemistry and tumor markers were studied. At autopsy, liver tumor weight and size, ascites, abdominal lymph nodes metastases, experimental peritoneal carcinomatosis index $(\mathrm{ePCl})$, and tumor-host body weight ratio were investigated. Immunohistochemical studies and western blotting were done to investigate key molecules involved in the mechanism of action.
\end{abstract}

Results: Compared with Control, both PDOX and DOX could similarly and significantly reduce liver tumor weight and tumor volume by over 40\%, ePCI values, retroperitoneal lymph node metastases and lung metastases and serum AFP levels $(P<0.05)$. The PDOX group had significantly higher WBC than the DOX group $(P<0.05)$, and higher PLT than Control $(P<0.05)$. Serum BUN and $\mathrm{Cr}$ levels were lower in the PDOX group than DOX and Control groups $(P<0.05)$. Compared with Control, DOX increased CK and CK-MB; while PDOX decreased CK compared with DOX $(P<0.05)$. Multiple spotty degenerative changes of the myocardium were observed in DOX-treated mice, but not in the Control and PDOX groups. PDOX could significantly reduce the Ki-67 positive rate of tumor cells, compared with DOX and Control groups. PDOX produced the effects at least via the ERK pathway.

Conclusion: Compared with DOX, PDOX may have better anti-metastatic efficacy and reduced side effects especially cardio-toxicities in this HCC model.

Keywords: Hepatocellular carcinoma, Molecular targeting therapy, Doxorubicin, PDOX, Metastases

\section{Background}

Hepatocellular carcinoma ( $\mathrm{HCC})$ is the fifth most frequent malignant tumors, and the third leading cause of cancer-related mortality in the world [1]. HCC patients are usually diagnosed when the tumor is in an advanced stage and lose the opportunity for curative surgery [2]. Other treatments including loco-regional or systemic

\footnotetext{
*Correspondence: liyansd2@163.com

${ }^{\dagger}$ Equal contributors

'Department of Oncology, Zhongnan Hospital of Wuhan University, Hubei Key Laboratory of Tumor Biological Behaviors \& Hubei Cancer Clinical Study Center, No 169 Donghu Road, Wuchang District, 430071 Wuhan, P.R. China Full list of author information is available at the end of the article
}

chemotherapy, fail mainly due to the chemoresistance of tumor and inability to endure treatment responses [3].

One of the most commonly used chemotherapy drugs for HCC is doxorubicin (DOX), but high doses of DOX result in severe toxicities, such as hematological, gastrointestinal, renal, hepatic toxicities, and particularly cardiac toxicities [4-6].

Increasing evidence supports the role of cathepsin B (Cat B) in tumor invasion and metastasis [7-9], including HCC progression [10]. Cat B expression is increased in many cancers at the mRNA, protein and activity levels, and closely related to invasive behavior of cancer [11].

\section{Biomed Central}


Therefore, Cat B could be a potential target for new drugs designed specifically against invading cancer cells.

To retain the therapeutic effect while reducing the toxicity of DOX, Dubowchik et al. [12-14] designed a smart prodrug of DOX, Ac-Phe-Lys-PABC-DOX (PDOX), in which a Cat B-specific dipeptide is introduced, along with a spacer PABC (para-aminobenzyloxycarbonyl) to increase the distance between dipeptide and DOX, so that the dipeptide can enter the Cat B' active site. As a result of this molecular re-structuring, the prodrug is inactive in blood circulation and normal tissues where little Cat B exists in the active form. When the prodrug reaches Cat B-enriched area such as the invasion front of cancer, the Phe-Lys dipeptide is cleaved by Cat B, exposing the PABC spacer that is then hydrolyzed spontaneously, releasing free DOX at the cancer invasion front. Thus this prodrug could exert cytotoxicity to invading cancer cells while protecting normal cells from excessive drug exposure, a strategy called passive targeted therapy.

In our previous animal model study, we investigated the activities and side effects of PDOX to treat peritoneal carcinomatosis (PC) from gastric cancer, which suggests that PDOX might be a promising new drug against cancer invasion [15]. Inspired by the initial results, we designed this study to further explore the treatment potential of this prodrug in a more aggressive and highly lethal orthotopic nude mice model of HCC.

\section{Materials and methods Agents and drugs}

The prodrug PDOX was synthesized according to the previously reported chemical process [12-14]. The molecular formula of PDOX is $\mathrm{C}_{52} \mathrm{H}_{59} \mathrm{~N}_{5} \mathrm{O}_{16} \cdot \mathrm{HCl}$, and the molecular weight is 1046.51 . In terms of equivalent mole content, $1.8 \mathrm{mg}$ PDOX is equivalent to $1 \mathrm{mg} \mathrm{DOX}$ (molecular weight 579.99). Doxorubicin for injection (DOX, Pharmacia, Milan, Italy, $10 \mathrm{mg}$ per vial) was obtained commercially.

\section{HCC cell lines and animal models}

Highly metastatic human HCC cell line HCCLM9 was used for animal model construction. This cell line was obtained by cloning culture, and 9 rounds of successive in vivo pulmonary metastases selections as described previously [16,17]. Cells were grown in RPMI 1640 medium (Mediatech, Manassas, VA) supplemented with $10 \%$ fetal bovine serum (FBS) and 1\% penicillin/streptomycin (Gibco, Carlsbad, CA). The cells were cultured in a humidified atmosphere at $37^{\circ} \mathrm{C}$ in $5 \% \mathrm{CO}_{2}$ and passaged if grown to $90 \%$ confluence.

\section{Orthotopic nude mice model of HCC and treatment}

Male athymic BALB/c nu/nu mice, 4-6 weeks old, were obtained from Beijing HFK Bio-Technology Co. Ltd [animal quality certificate No. SCXK (jing) 290004] and housed in specific pathogen-free (SPF) condition at the Animal Experiment Center of Wuhan University. All animal experiments were carried out in accordance with the guidelines and approved protocols of the University of Wuhan Animal Experiment Center Institutional Animal Care and Use Committee (Permit Number 00024763).

Nude mice model with spontaneous pulmonary metastasis was established as described previously [17]. Briefly, HCCLM9 cells $\left(5 \times 10^{6}\right.$ cells each) in $0.1 \mathrm{ml}$ phosphate buffered saline (PBS) were injected subcutaneously into the left upper flank of 2 nude mice. The subcutaneous tumors were removed when they reached $8 \mathrm{~mm}$ in diameter, and minced into pieces $\left(1 \mathrm{~mm}^{3}\right)$ to perform orthotopic transplantation into livers of nude mice $(\mathrm{n}=$ 33). On day 8 after model establishment, the mice were randomized into Control group $(n=10)$, DOX group $(\mathrm{n}=11)$, and PDOX group $(\mathrm{n}=12)$, and treated as illustrated in the flow chart of Figure 1 (Figure 1A).

In our previous study [15], we found that by intraperitoneal injection, PDOX could be safely administered at twice the dose of DOX on a molar basis. Therefore, we increased the doses of both DOX $(4 \mathrm{mg} / \mathrm{kg})$ and PDOX $(21.6 \mathrm{mg} / \mathrm{kg})$ in this study, in which the PDOX dose was 3 times that of DOX on a molar basis.

The behaviors and general conditions were monitored daily, and body weights of animals were recorded twice a week. On d 36 post inoculation, the mice were anesthetized by peritoneal injection of $3 \%$ phenobarbital chloride, and then subjected to magnetic resonance image (MRI) study to measure the liver tumor size (Bruker Biospec $4.7 \mathrm{~T} / 30$, Germany). The peripheral blood was obtained for routine blood tests and biochemistry studies. Tumor tissues and major organs including the heart, the liver, the lungs and any other suspected organs were collected, fixed with $4 \%$ paraformaldehyde and embedded in paraffin for pathological and immunohistochemistry studies. In addition, fresh tumor tissues were obtained for western blotting studies as detailed below.

\section{Experimental peritoneal carcinomatosis index (ePCl)}

An experimental peritoneal carcinomatosis index (ePCI) system was developed to evaluate the efficacy that took into consideration of tumor nodule sizes, distributions, and the characteristics of ascites. In this system, the abdominal cavity of the mouse was divided into 4 regions: region I, subdiaphragm; region II, the liver, spleen, stomach, and affiliated ligaments; region III, the small intestine, colon, mesenterium, and abdominal wall; and region IV, the pelvic cavity, urogenital system, and rectum. The detailed scoring criteria were modified from a similar reporting system on a rat peritoneal carcinomatosis model [18] and set forth in our previous report [15]. 


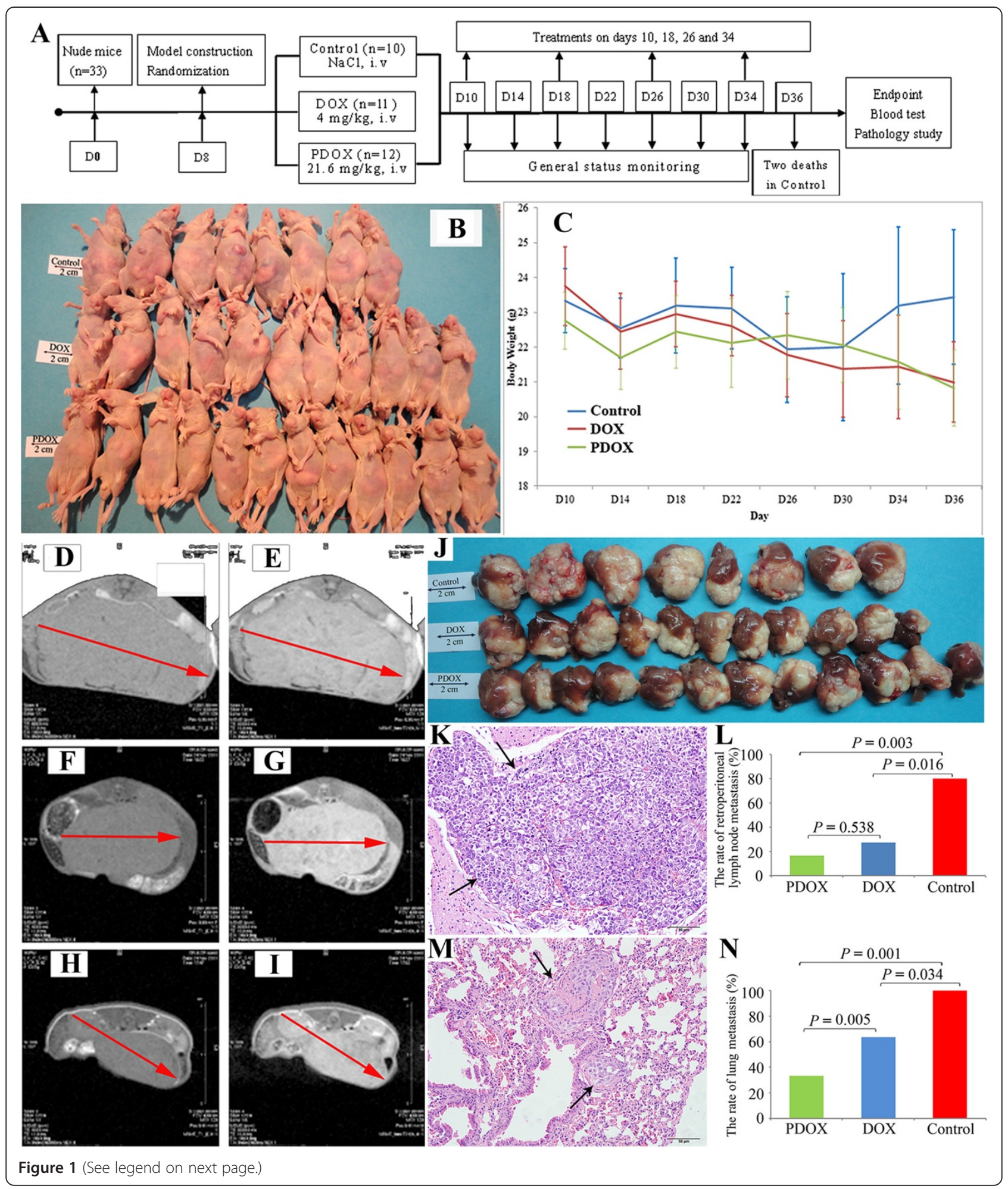


(See figure on previous page.)

Figure 1 The study procedure and major results. A: The flow chart of this study. Nude mice were kept for $3 \mathrm{~d}$ of adaptation, and orthotopic model of human HCC was constructed as described in the Materials and Methods section. On d 8, the animals were randomized into 3 groups, and treated by tail vein injection of normal saline, DOX and PDOX, respectively. At the study endpoint, all animals were euthanized, and detailed pathological studies. B: The general status of animals appeared best in the PDOX, better in DOX, and worst in Control that were only 8 animals because 2 nude mice died before the study endpoint due to excessive tumor burden. $\mathbf{C}$ : The body weight curves at different time points show similar changes in the PDOX and DOX. In Control, there was a progressive increase on the last $6 \mathrm{~d}$, due to accelerated tumor growth and increased ascites. D-I: Representative MRI scans of the liver tumors in Control (D for T1 and $\mathbf{E}$ for T2), the DOX (F for T1 and $\mathbf{G}$ for T2) and the PDOX ( $\mathbf{H}$ for T1 and I for T2) showed marked differences in tumor size. J: Both PDOX and DOX had similar and significant liver tumor growth inhibition compared with Control that 2 animals died on the morning of $d$ 36, therefore only 8 tumors were available in the final picture presentation. $\mathbf{K} \& \mathbf{L}$ : PDOX and DOX treatments significantly reduced retroperitoneal lymph nodes metastases (arrows) compared with Control. $\mathbf{M} \& \mathbf{N}$ : The lung metastases (arrows) were also significantly reduced in the PDOX and DOX, and PDOX had greater inhibitory effect on lung metastases than. DOX. D: day; T1: T1-weighted image; T2: T2-weighted image; $\mathbf{K} \& \mathbf{M}$ : hematoxylin and eosin stain, $200 \times$, scale bar $=50 \mu m$.

\section{Immunohistochemistry study}

Tumor tissues obtained from animals of 3 groups were subjected to immunohistochemistry to detect the expressions of Cat B, Ki-67, CD34, VEGF, E-cadherin and D2-40, according to our previously reported procedures [15]. The primary antibodies for Cat B (Cat No 3190100, BioVision, CA, USA, dilution 1:200), Ki-67 (MAB0129, Maxim-Bio Co, CHN, Working solution), CD34 (BA0532, WuHan Boster Bio-Engineering Co, CHN, dilution 1:100), VEGF (RB-9031, Maxim-Bio Co, CHN, Working solution), E-cadherin (MAB-0589, Maxim-Bio Co, CHN, Working solution) and D2-40 (AM0103, Ascend Biotechnology Co, CHN, Working solution) were prepared and incubated with the slides for $2 \mathrm{~h}$ in a moist chamber. After a new cycle of washes, the slides were again placed in a moist chamber for 30-minute incubation with a biotinylated secondary antibody and biotinperoxidase complex (Biogenex, SF, USA, Working solution). The color of immunoperoxidase reaction was achieved by immersion for $5 \mathrm{~min}$ in a solution containing the DAB chromogen (3,5-diamino-benzidine tetra-hydrochloride) and counterstained with hematoxylin for $2 \mathrm{~min}$. The slides were observed under the microscope.

For the evaluation of IHC results, positive cells were stained brownish granules in the cell membrane, cytoplasm or nucleus. In all cases, cytoplasmic Cat B expression was scaled as moderate and strong expression. Ki-67 expressed in the nucleus. VEGF positive cells were stained both in the nucleus and cytoplasm. The expression of E-cadherin mainly existed in cell membrane and cytoplasm. CD34 and D2-40 positive cells were stained in cytoplasm. Ten fields in each slide were selected randomly and observed at a magnification of $\times 200$. The expression of Ki-67 was evaluated according to positive rate. The positive expression of CD34 and D2-40 was evaluated according to microvessel density (MVD) and lymphatic microvessel density (LMVD).

\section{Western blotting study}

Fresh tumor tissues in RIPA lysis buffer containing $1 \mu \mathrm{g} /$ ml PMSF, $1 \times$ Cocktail, were manually homogenized on ice using a glass homogenizer, then centrifuged at $3000 \mathrm{~g}$ for $10 \mathrm{~min}$ to remove cellular and nuclear debris. The protein concentration was determined using a BCA Assay kit (Biyuntian).

To determine the expressions of p-ERK1/2, ERK $1 / 2$, Bcl-2, caspase- 3 , and $\beta$-actin using western blotting, $100 \mu \mathrm{g}$ total proteins were separated by SDS-polyacrylamide gel electrophoresis (4\% stacking and 10\% separating gels) and then transferred overnight onto PVDF membranes, which were blocked with 5\% skimmed milk in $0.01 \mathrm{M}$ phosphate buffer solution (PBS) containing $0.05 \%(\mathrm{v} / \mathrm{v})$ Tween. Next, they were immunoblotted with a rabbit anti-human p-ERK (4370, CST, MA, USA, dilution 1:1000), rabbit anti- human ERK (4695, CST, MA, USA, dilution 1:1000), rabbit anti-human Bcl-2 (2870, CST, MA, USA, dilution 1:1000), rabbit anti-human caspase-3 (9665, CST, MA, USA, dilution 1:1000), mouse antihuman caspase-9 (9508, CST, MA, USA, dilution 1:1000), and rabbit anti-human $\beta$-actin (Santa Cruz, CA, USA, dilution 1:1000) for $3 \mathrm{~h}$. Blots were then incubated with a peroxidase-conjugated sheep anti-rabbit IgG (Santa Cruz, CA, USA, dilution 1:8000) or sheep anti-mouse IgG (Santa Cruz, CA, USA, dilution 1:8000) for $2 \mathrm{~h}$ and developed using chemiluminescent detection with a Supersignal West Pico assay kit (Thermo, IL, USA) and autoradiography film.

\section{Blood tests and biochemistries}

On d 36, animals were euthanized, and blood was obtained for routine studies, including peripheral blood profiles by Sysmex KX-21 automated hematology analyzer (Sysmex, Kobe, Japan); liver function parameters alanine aminotransferase (ALT), aspartate aminotransferase (AST), gamma-glutamyl transpeptidase (GGT), total bilirubin levels (TBIL), and direct bilirubin (DBIL) levels; renal function parameters blood urea nitrogen (BUN) and 
Table 1 Effects on tumor growth and metastases

\begin{tabular}{|c|c|c|c|c|c|c|}
\hline \multirow[t]{3}{*}{ Items } & \multicolumn{5}{|c|}{ Treatment groups } & \multirow[t]{3}{*}{$P$ value } \\
\hline & \multirow[t]{2}{*}{ Control $(n=10)$} & \multicolumn{2}{|c|}{$\operatorname{DOX}(n=11)$} & \multicolumn{2}{|c|}{ PDOX $(n=12)$} & \\
\hline & & Value & Inhibition ratio ${ }^{\text {it }}$ & Value & Inhibition ratio ${ }^{\text {th }}$ & \\
\hline \multicolumn{7}{|l|}{ Liver tumor growth } \\
\hline Tumor weight (mg) & $6657.4 \pm 1312.9^{*}, \S$ & $3860.0 \pm 1023.6$ & $42.0 \%$ & $3757.6 \pm 603.5$ & $43.6 \%$ & $\begin{array}{l}{ }^{*}<0.001 \text { vs DOX } \\
{ }^{\S}<0.001 \text { vs PDOX }\end{array}$ \\
\hline Tumor volume $\left(\mathrm{mm}^{3}\right)$ & $4965.2 \pm 2112.4^{*}, \$$ & $2526.9 \pm 1360.1$ & $49.1 \%$ & $2313.2 \pm 675.7$ & $53.4 \%$ & $\begin{array}{l}{ }^{*}<0.05 \text { vs DOX } \\
\S<0.001 \text { vs PDOX }\end{array}$ \\
\hline TW/BW ratio & $27.94 \pm 4.10^{*}, \$$ & $18.28 \pm 4.12$ & $34.5 \%$ & $18.10 \pm 3.18$ & $35.2 \%$ & $\begin{array}{l}{ }^{*}<0.001 \text { vs DOX } \\
s^{\S}<0.001 \text { vs PDOX }\end{array}$ \\
\hline AFP level (ng/mL) & $97.27 \pm 34.22^{*}, \S$ & $24.69 \pm 12.09$ & $74.6 \%$ & $22.31 \pm 13.42$ & $77.1 \%$ & $\begin{array}{l}{ }^{*}<0.001 \text { vs DOX } \\
s<0.001 \text { vs PDOX }\end{array}$ \\
\hline $\mathrm{ePCl}$ & $9 \pm 2^{*}, \$$ & $6 \pm 2$ & $33.3 \%$ & $6 \pm 2$ & $33.3 \%$ & $\begin{array}{l}{ }^{*}<0.05 \text { vs DOX } \\
\S^{<}<0.05 \text { vs PDOX }\end{array}$ \\
\hline \multicolumn{7}{|l|}{ Tumor metastases } \\
\hline Mediastinal LN meta & $70.0 \%(7 / 10)$ & $63.6 \%(7 / 11)$ & $9.1 \%$ & $33.3 \%(4 / 12)$ & $52.4 \%$ & $>0.05$ \\
\hline Lung meta & $100 \%(10 / 10)^{*}, \$$ & $63.6 \%(7 / 11)^{\#}$ & $36.4 \%$ & $33.3 \%(4 / 12)$ & $66.7 \%$ & $\begin{array}{l}{ }^{*}<0.05 \text { vs DOX } \\
\S^{\S}<0.05 \text { vs PDOX } \\
{ }^{\#}<0.05 \text { vs PDOX }\end{array}$ \\
\hline Diaphragm meta & $90 \%(9 / 10)$ & $72.7 \%(8 / 11)$ & $19.2 \%$ & $50 \%(6 / 12)$ & $44.4 \%$ & $>0.05$ \\
\hline Intrahepatic meta & $30 \%(3 / 10)$ & $27.3 \%(3 / 11)$ & $9.0 \%$ & $50 \%(6 / 12)$ & $-66.7 \%$ & $>0.05$ \\
\hline Spleen ligament meta & $70 \%(7 / 10)$ & $36.4 \%(4 / 11)$ & $48.0 \%$ & $41.7 \%(5 / 12)$ & $40.4 \%$ & $>0.05$ \\
\hline Heptogastric ligament meta & $90 \%(9 / 10)$ & $90.9 \%(10 / 11)$ & $-1.0 \%$ & $83.3 \%(10 / 12)$ & $7.4 \%$ & $>0.05$ \\
\hline Renal ligament meta & $40.0 \%(4 / 10)$ & $36.4 \%(4 / 11)$ & $9.0 \%$ & $58.3 \%(7 / 12)$ & $-45.8 \%$ & $>0.05$ \\
\hline Adrenal meta & $10.0 \%(1 / 10)$ & $0.0 \%(0 / 11)$ & $100 \%$ & $0.0 \%(0 / 12)$ & $100 \%$ & $>0.05$ \\
\hline Mesenteric meta & $90.0 \%(9 / 10)$ & $81.8 \%(9 / 11)$ & $9.1 \%$ & $58.3 \%(7 / 12)$ & $35.2 \%$ & $>0.05$ \\
\hline Retroperitoneal LN meta & $80.0 \%(8 / 10)^{*}, \$$ & $27.3 \%(3 / 11)$ & $65.9 \%$ & $16.7 \%(2 / 12)$ & $79.1 \%$ & $\begin{array}{l}{ }^{*}<0.05 \text { vs DOX } \\
{ }^{<}<0.05 \text { vs PDOX }\end{array}$ \\
\hline Abdominal wall meta & $80.0 \%(8 / 10)$ & $45.5 \%(5 / 11)$ & $43.1 \%$ & $66.7 \%(8 / 12)$ & $16.6 \%$ & $>0.05$ \\
\hline Bloody ascites & $50.0 \%(5 / 10)$ & $9.1 \%(1 / 11)$ & $81.8 \%$ & $25.0 \%(3 / 12)$ & $50.0 \%$ & $>0.05$ \\
\hline
\end{tabular}

TW: tumor weight; BW: body weight; AFP: alpha-fetoprotein; ePCl: experimental peritoneal carcinomatosis index; $L N$ : lymph nodes; Meta: metastasis.

${ }^{2}$ All the inhibition ratios were compared with the Control group. ${ }^{*}, \S$, \#: denote statistical significances for comparisons of the same item in different groups, as clearly described in the $\mathrm{P}$ value column.

creatinine $(\mathrm{Cr})$ levels; cardiac function parameters creatine kinase $(\mathrm{CK})$, creatine kinase-MB (CK-MB) and lactate dehydrogenase (LDH) levels; electrolytes $\left(\mathrm{K}^{+}, \mathrm{Na}^{+}, \mathrm{Ca}^{2+}\right.$, $\mathrm{Mg}^{2+}$ and $\mathrm{Cl}^{-}$) and serum alpha fetoprotein (AFP) levels; all by Aeroset Clinical Chemistry Analyzer (Abbott Laboratories, IL, USA).

\section{Statistical analysis}

All data were analyzed using the statistical software of SPSS 13.0 for Windows (SPSS Inc, Chicago, USA). The differences in body weights, liver tumor weights, and the expression of Ki-67, CD34 and D2-40 among different groups were tested by one-way ANOVA. The differences of Cat B, VEGF and E-cadherin were analyzed by the chi-square $\left(X^{2}\right)$ test. $P$-value $<0.05$ was considered as statistically significant.

\section{Results}

PDOX had better effects on general status and similar inhibitory effects on liver tumor growth and loco-regional metastases

After tumor inoculation into the liver, the animals in the DOX and PDOX groups showed slight and progressive body weight decreases till the study endpoint. The general status of animals appeared better in the PDOX group than the DOX group, which in turn was better than Control group (Figure 1B). In the Control group, the animals showed body weight increases from $d 30$ to d 36, mainly due to excessive liver tumor and ascites (Figure 1C).

Prominent liver tumors were observed in all animals, and representative MRI abdominal scan of liver tumors were shown (Figure 1D to 1I). At the study endpoint, the tumor weights were $6657.4 \pm 1312.9 \mathrm{mg}$ in the 
Table 2 Immunohistochemical analysis

\begin{tabular}{lllll}
\hline Items & \multicolumn{3}{c}{ Treatment groups } & P value \\
\cline { 2 - 4 } & Control $(\mathbf{n}=\mathbf{1 0})$ & $\mathbf{D O X}(\mathbf{n}=\mathbf{1 1})$ & PDOX $(\mathbf{n}=\mathbf{1 2})$ & \\
\hline Cat B & $100 \%(10 / 10)$ & $10 \%(11 / 11)$ & $100 \%(12 / 12)$ & $P>0.05$ \\
VEGF & $100 \%(10 / 10)$ & $90.9 \%(10 / 11)$ & $83.3 \%(10 / 12)$ & $P>0.05$ \\
E-cadherin & $100 \%(10 / 10)$ & $90.9 \%(10 / 11)$ & $100 \%(12 / 12)$ & $P>0.05$ \\
Ki-67 & $77.1 \pm 7.8 \% *^{*}$ & $72.3 \pm 4.9 \%{ }^{\#}$ & $61.6 \pm 14.6 \%$ & $* P<0.05$, Control vs PDOX ${ }^{\#} P<0.05$, DOX vs PDOX \\
CD34 & $47.2(21.4-70.0)$ & $60.9(37.0-91.2)$ & $55.6(22.2-80.2)$ & $P>0.05$ \\
D2-40 & $0.5(0.0-3.2)$ & $1.8(0.0-8.4)$ & $1.8(0.0-5.8)$ & $P>0.05$ \\
\hline
\end{tabular}

Notes: Cat B, VEGF and E-cadherin were evaluated according to the percentage of positive expression (number of animals with positive expression $\div$ number of all animals in the group $\times 100 \%$ ). The expression of Ki-67 was evaluated according to positive rate in every animal. The positive expression of CD34 and D2-40 was evaluated according to microvessel density (MVD) and lymphatic microvessel density (LMVD).

* \#: denote statistical significances for comparisons of the same item in different groups, as clearly described in the P value column.

Control group, $3860.0 \pm 1023.6 \mathrm{mg}$ in the DOX group, and $3757.6 \pm 603.5 \mathrm{mg}$ in the PDOX group $(P<0.001$, Control vs PDOX; $P<0.001$, Control vs DOX; $P>0.05$, DOX vs PDOX). Compared with Control, PDOX and DOX treatments reduced tumor weights by $43.6 \%$ and $42.0 \%$, respectively. Similarly, PDOX and DOX treatments reduced tumor volumes by $53.4 \%$ and $49.1 \%$, respectively $(P<0.01$, DOX/PDOX $v s$ Control) (Figure 1), Table 1). The tumor-weight to body-weight ratio was also significantly reduced from $27.94 \%$ in the Control group to $18.28 \%$ in the DOX group and $18.10 \%$ in the PDOX group $(P<0.001$, DOX/PDOX vs Control). The serum AFP level was reduced from 97.27 \pm $34.22 \mathrm{ng} / \mathrm{mL}$ in the Control group to $24.69 \pm 12.09$ $\mathrm{ng} / \mathrm{mL}$ in the DOX group and $22.31 \pm 13.42 \mathrm{ng} / \mathrm{mL}$ in the PDOX group $(P<0.001$, DOX/PDOX vs Control $)$ (Table 1).

In addition to liver tumor reduction, the loco-regional metastases were also investigated. We used the ePCI score system to evaluate the peritoneal metastases of this model. The ePCI was reduced from $9 \pm 2$ in the Control group to $6 \pm 2$ in the DOX group and $6 \pm 2$ in the PDOX group $(P<0.05$, DOX/PDOX vs Control). Another significant effect was observed on retroperitoneal lymph node metastases, which occurred in $80.0 \%(8 / 10), 27.3 \%$ $(3 / 11)$ and $16.7 \%(2 / 12)$ of animals, respectively, in the Control, DOX and PDOX groups $(P<0.05$, DOX/PDOX vs Control) (Figure $1 \mathrm{~K}$ and $1 \mathrm{~L}$, Table 1 ).

\section{PDOX had better inhibitory effects on lung metastases than DOX}

Treatment effects on distant metastases were also studied. The rates of animals with lung metastases were reduced from $100.0 \%(10 / 10)$ in the Control group to $63.6 \%(7 / 11)$ in the DOX group and $33.3 \%(4 / 12)$ in the PDOX group $(P<0.05$, Control $v s$ DOX; $P<0.05$, Control vs PDOX; $P<0.05$, DOX $v s$ PDOX) (Figure $1 \mathrm{M}$ and $1 \mathrm{~N}$, Table 1).

\section{PDOX had higher inhibitory effect on tumor proliferation than DOX}

IHC studies were performed to investigate the expression of major cancer molecules possibly affected by the treatments. As shown in Table 2 and Figure 2, positive cytoplasmic Cat B expression was observed in all tumors from the 3 groups. Ki-67 positive rates were $77.1 \pm 7.8 \%$ in the Control group, $72.3 \pm 4.9 \%$ in the DOX group, and $61.6 \pm 14.6 \%$ in the PDOX group $(P>0.05$, Control vs DOX; $P<0.05$, Control vs PDOX; $P<0.05$, DOX vs PDOX). The median (range) MVD values of CD34 were 47.2 (21.4-70.0) in the Control group, 60.9 (37.0-91.2) in the DOX group, and $55.6(22.2-80.2)$ in the PDOX group, respectively $(P>0.05)$. The VEGF positive rate was not statistically different among the 3 groups $(P>$ 0.05). Similarly, there was no statistical difference in the expression of E-cadherin among the 3 groups $(P>$ 0.05 ). The median (range) values of LMVD designated as D2-40 positive expression were $0.5(0.0-3.2), 1.8$ $(0.0-8.4)$ and $1.8(0.0-5.8)$ in the Control, DOX and PDOX groups, respectively $(P>0.05)$.

\section{PDOX had less hematological and biochemical toxicities than DOX}

The hematological and non-hematological toxicities were studied (Table 3). In peripheral blood routine, the white blood cells levels in PDOX mice were higher than DOX mice (1.98-folds, $P<0.05)$. The platelet levels were higher in the PDOX group $(1.67$-folds, $P<0.05)$ and the DOX group (1.59-folds, $P>0.05$ ) compared with Control. There were no differences in red blood cells and hemoglobin levels among the 3 groups.

In terms of liver functions, compared with Control, DOX and PDOX caused significant reduction in GGT and AST levels $(P<0.05$, DOX/PDOX vs Control $)$ (Table 3 ). There were no statistically significant differences in AST, TBIL and DBIL levels among the 3 groups. 


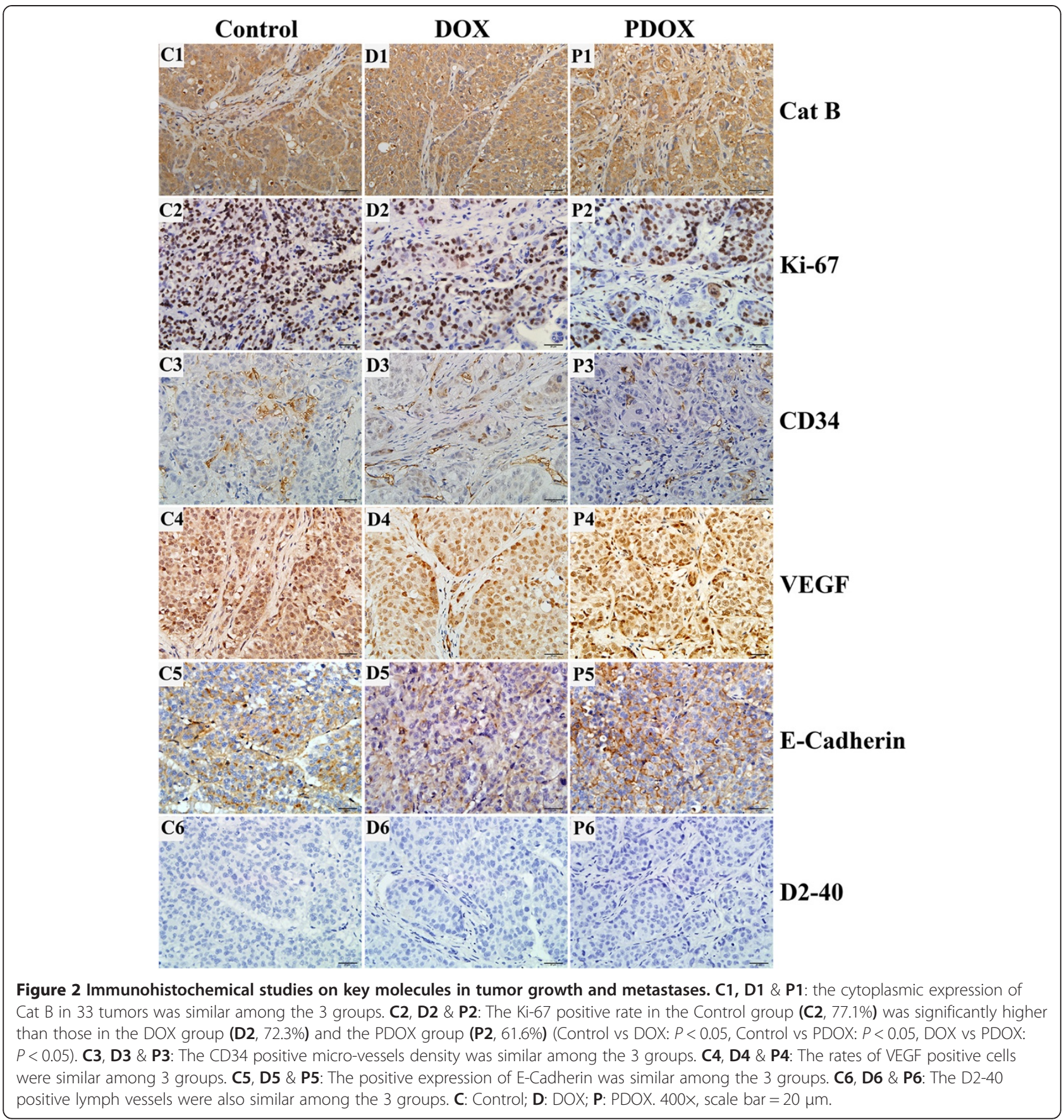

In terms of renal functions, compared with Control, both DOX and PDOX resulted in significant reduction in serum BUN levels $(P<0.001$, Control vs DOX; $P<$ 0.05, Control vs PDOX), and BUN levels in the PDOX group were also significantly lower than those in the DOX group $(P<0.05)$. Furthermore, the serum $\mathrm{Cr}$ levels in the PDOX group were much lower than those of the Control and DOX groups $(P<0.05$, PDOX $v$ s Control; $P<0.05$, PDOX vs DOX) (Table 3).
Electrolytes results demonstrated that $\mathrm{Cl}^{-}$was reduced in PDOX compared with Control group $(P<0.05)$; But $\mathrm{Ca}^{2+}$ was increased in PDOX compared with the Control and DOX groups $(P<0.05$, PDOX vs Control; $P<0.05$, PDOX vs DOX) (Table 3).

\section{PDOX had less cardio-toxicity than DOX}

Cardiac functions demonstrated that both DOX and PDOX significantly decreased LDH compared with Control group 
Table 3 Routine blood tests and biochemistry (expressed as mean \pm SD)

\begin{tabular}{|c|c|c|c|c|}
\hline \multirow[t]{2}{*}{ Items } & \multicolumn{3}{|c|}{ Treatment groups } & \multirow[t]{2}{*}{$P$ value } \\
\hline & Control $(n=8)^{\sqrt[2]{2}}$ & $\operatorname{DOX}(n=11)$ & PDOX $(n=12)$ & \\
\hline \multicolumn{5}{|c|}{ Peripheral blood routine } \\
\hline $\mathrm{RBC}\left(\times 10^{12} / \mathrm{L}\right)$ & $8.06 \pm 3.50$ & $9.59 \pm 0.65$ & $9.52 \pm 1.47$ & $>0.05$ \\
\hline $\mathrm{HGB}(\mathrm{g} / \mathrm{L})$ & $139.00 \pm 9.82$ & $141.18 \pm 9.62$ & $138.83 \pm 19.52$ & $>0.05$ \\
\hline WBC $\left(\times 10^{9} / L\right)$ & $3.25 \pm 1.50$ & $2.71 \pm 1.00^{*}$ & $5.37 \pm 3.31^{*}$ & $<0.05$ \\
\hline $\operatorname{PLT}\left(\times 10^{9} / \mathrm{L}\right)$ & $385.67 \pm 102.72^{*}, \S$ & $611.36 \pm 176.40$ & $644.33 \pm 293.34$ & $\begin{array}{l}{ }^{*}<0.05 \text { vs DOX } \\
{ }^{\S}<0.05 \text { vs PDOX }\end{array}$ \\
\hline \multicolumn{5}{|l|}{ Liver functions } \\
\hline AST (U/L) & $489.01 \pm 95.85^{*}$ & $338.42 \pm 75.47^{\S}$ & $444.64 \pm 114.01$ & $\begin{array}{l}{ }^{*}<0.05 \text { vs DOX } \\
{ }^{\S}<0.05 \text { vs PDOX }\end{array}$ \\
\hline $\operatorname{ALT}(U / L)$ & $172.83 \pm 52.26$ & $219.56 \pm 165.57$ & $216.11 \pm 153.50$ & $>0.05$ \\
\hline TBIL $(\mu \mathrm{mol} / \mathrm{L})$ & $3.51 \pm 0.42$ & $3.40 \pm 0.50$ & $3.59 \pm 0.67$ & $>0.05$ \\
\hline DBIL $(\mu \mathrm{mol} / \mathrm{L})$ & $3.21 \pm 0.10$ & $3.17 \pm 0.11$ & $3.19 \pm 0.16$ & $>0.05$ \\
\hline GGT (U/L) & $30.28 \pm 10.65 *$, § & $15.75 \pm 6.13$ & $18.43 \pm 11.06$ & $\begin{array}{l}{ }^{*}<0.05 \text { vs DOX } \\
{ }^{\S}<0.05 \text { vs PDOX }\end{array}$ \\
\hline \multicolumn{5}{|l|}{ Renal functions } \\
\hline BUN (mmol/L) & $15.83 \pm 1.72^{*}, \S$ & $11.99 \pm 3.33^{\#}$ & $9.29 \pm 2.40$ & $\begin{array}{l}{ }^{*}<0.05 \text { vs DOX } \\
\S<0.001 \text { vs PDOX } \\
{ }^{\#}<0.05 \text { vs PDOX }\end{array}$ \\
\hline $\mathrm{Cr}(\mu \mathrm{mol} / \mathrm{L})$ & $41.73 \pm 3.85^{*}$ & $43.37 \pm 4.95^{\S}$ & $34.76 \pm 7.67$ & $\begin{array}{l}{ }^{*}<0.05 \text { vs PDOX } \\
{ }^{\S}<0.05 \text { vs PDOX }\end{array}$ \\
\hline \multicolumn{5}{|l|}{ Electrolytes } \\
\hline $\mathrm{K}^{+}(\mathrm{mmol} / \mathrm{L})$ & $6.56 \pm 0.60$ & $6.58 \pm 0.36$ & $6.81 \pm 0.59$ & $>0.05$ \\
\hline $\mathrm{Na}^{+}(\mathrm{mmol} / \mathrm{L})$ & $160.82 \pm 4.04$ & $158.53 \pm 2.21$ & $158.50 \pm 2.54$ & $>0.05$ \\
\hline $\mathrm{Cl}^{-}(\mathrm{mmol} / \mathrm{L})$ & $114.88 \pm 1.86^{*}$ & $113.23 \pm 2.66$ & $112.42 \pm 1.58^{*}$ & $<0.05$ \\
\hline $\mathrm{Ca}^{2+}(\mathrm{mmol} / \mathrm{L})$ & $2.25 \pm 0.10^{*}$ & $2.31 \pm 0.08^{\S}$ & $2.38 \pm 0.08$ & $\begin{array}{l}{ }^{*}<0.05 \text { vs PDOX } \\
{ }^{\S}<0.05 \text { vs PDOX }\end{array}$ \\
\hline $\mathrm{Mg}^{2+}(\mathrm{mmol} / \mathrm{L})$ & $1.15 \pm 0.09$ & $1.23 \pm 0.18$ & $1.15 \pm 0.34$ & $>0.05$ \\
\hline
\end{tabular}

Note: Two nude mice died before the study endpoint, leaving only 8 mice available for blood test of routine and biochemistry parameters. *, $\$$, \#: denote statistical significances for comparisons of the same item in different groups, as clearly described in the $P$ value column.

WBC: white blood cell; NEUT: neutrophil; RBC: red blood cell; HGB: hemoglobin; PLT: platelet; LYM: lymphocyte; PLT: platelet count; $A L T$ : alanine transaminase; $A S T$ : aspartate aminotransferase; TBIL: total bilirubin; DBIL: direct bilirubin; GGT: $\gamma$-glutamyltransferase; $C r$ : creatinine; $B U N$ : Blood urea nitrogen; $C K$ : creatine kinase; $C K-M B$ : creatine kinase-myoglobin; $L D H$ : lactic dehydrogenase.

$(P<0.05$, DOX/PDOX vs Control), but there were no differences between the DOX and PDOX groups. Compared with Control, DOX increased CK and CK-MB levels, although the differences didn't reach the statistical significance. On the other hand, PDOX significantly decreased CK, compared with DOX $(P<0.05)$ (Figure 3A, $3 \mathrm{~B}$ and $3 \mathrm{C})$.

Histopathological study revealed multiple spotty degenerative changes in the myocardium in DOX-treated mice (Figure 3F and 3G). There were no observable histopathological changes in both Control and PDOX groups (Figure 3D, 3E, 3H and $3 \mathrm{I}$ ).

\section{PDOX produced the effect at least by the ERK pathway} To investigate the mechanism of PDOX producing effects, we used western blotting to study the expression of ERK, p-ERK, BCL-2, caspase-3, and caspase-9. The results showed that PDOX and DOX reduced ERK phosphorylation, decreased BCL-2 expression, and activated caspase- 3 and caspase-9 (Figure 4).

\section{Discussion}

Major treatment modalities for HCC are surgery, chemotherapy, regional therapies such as radiofrequency ablation, transarterial chemoembolization (TACE) and molecular targeting therapies. In either systemic chemotherapy or TACE, DOX is one of the most commonly used drugs with proven efficacy, but has serious side effects. Among 475 patients who received DOX in various studies, a $16 \%$ response rate was documented, with a median survival time of 3-4 months [19]. Significant grade 3 or above hematologic and gastrointestinal toxicities were encountered in patients treated with DOX, including neutropenia (63\%), febrile neutropenia (17\%), thrombocytopenia (24\%), 

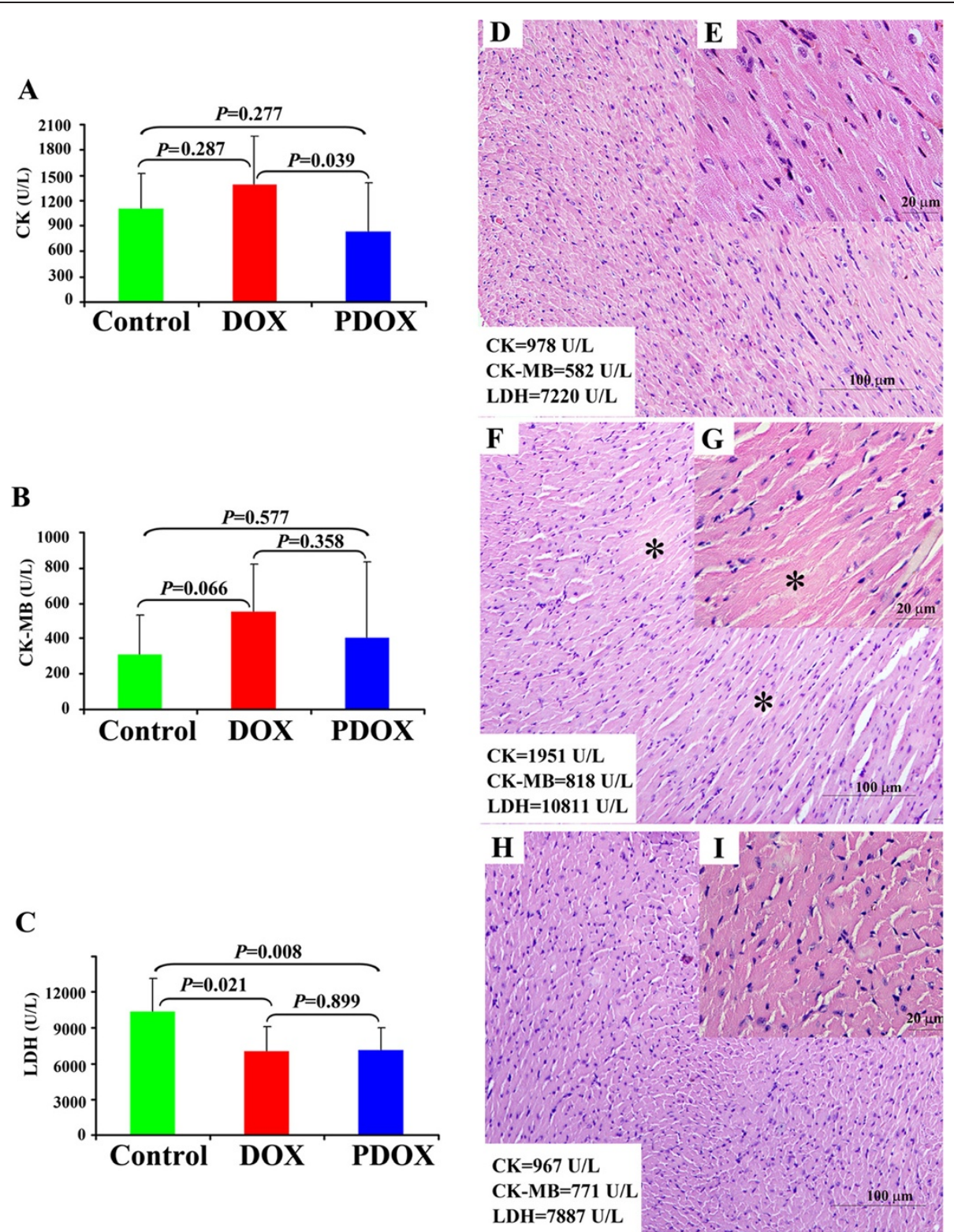

Figure 3 The cardio-toxicities of animals in the $\mathbf{3}$ groups. A: Compared with Control, DOX increased CK levels but without statistical significance, while PDOX significantly decreased CK levels compared with DOX $(P<0.05)$. B: Compared with Control, DOX increased CK-MB levels without significant difference, but PDOX did not increase CK-MB levels. C: Both DOX and PDOX significantly decreased LDH compared with Control group $(P<0.05$ and $P<0.05)$. D \& E: There were no observable histopathological changes in the myocardium of the Control mice. $\mathbf{F} \& \mathbf{G}$ Multiple spotty degenerative changes were observed in the myocardium of the DOX-treated mice. $\mathbf{H} \& \mathbf{I}$ : There were no observable histopathological changes in the myocardium of the PDOX-treated mice.

elevation of transaminases (13\%), and diarrhea (7\%) [20]. Therefore, it is imperative to modify DOX molecules in order to reduce its toxicities while maintaining its efficacy.

To fulfill this unmet clinical demand, Dubowchik et al. [12-14] designed a smart prodrug of DOX, which targets the active invading cancer cells and spares normal cells, because cancer cells, but not normal ones, secrete extracellular Cat B. It had previously been determined [12] that PDOX is stable in human and mouse blood. The major question, then, was whether PDOX reaches the tumor, is cleaved rapidly by Cat B, and the free DOX then enters into the cancer cells before it diffuses away. We now report, in this and in our previous paper [15], that PDOX indeed displays antitumor power at least equal to that of free DOX.

In this experimental study on a highly metastatic animal model of HCC, PDOX showed tumor inhibition similar to that of DOX, but significantly reduced toxicity 


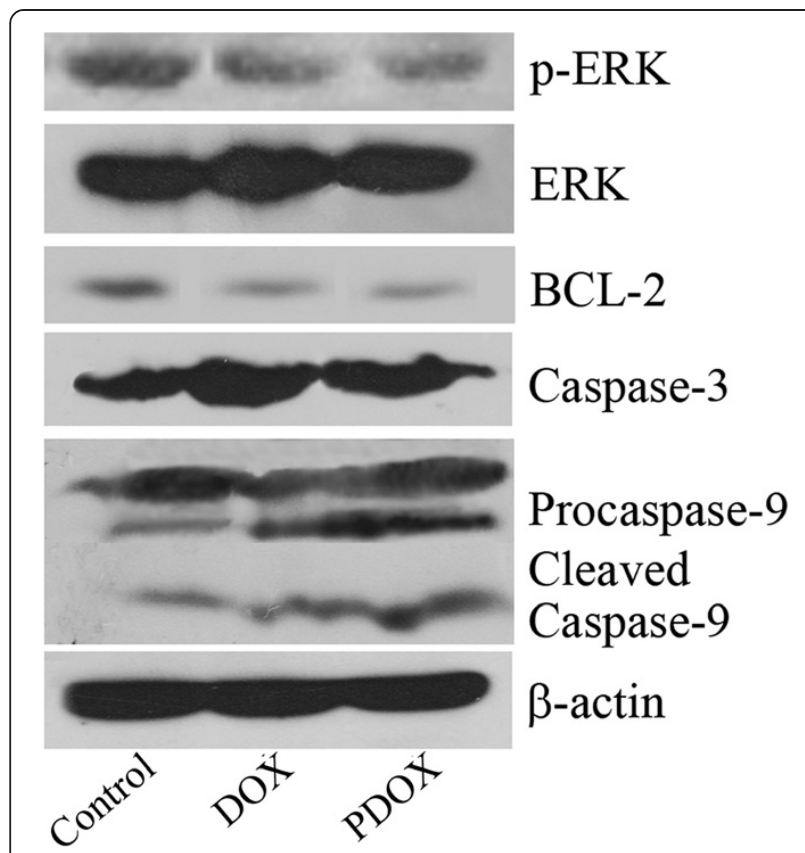

Figure 4 Western blotting showed that compared with Control, PDOX and DOX reduced ERK phosphorylation, decreased BCL-2 expression, and increased caspase- 3 and caspase- 9 activation.

profiles. Thus PDOX not only reaches the tumor as easily as free DOX, but is efficiently cleaved to free DOX there. In terms of liver tumor reduction, both PDOX and DOX treatments resulted over $40 \%$ of tumor growth inhibition. The general status of animals at the study endpoint also appeared better in the PDOX group. These results suggest that PDOX is at least as effective as DOX in this animal model.

Another possibility is that metastatic cells, which display more Cat B than those in the primary tumor [11], might have increased sensitivity to PDOX, and indeed they do. The primary tumor inhibition ratios relative to control by PDOX and DOX were $43.6 \%$ and $42.0 \%$. In contrast, the metastases inhibition ratios relative to control by PDOX and DOX were 52.4\% and 9.1\% for mediastinal lymph nodes metastasis, $66.7 \%$ and $36.4 \%$ for lung metastasis, $44.4 \%$ and $19.2 \%$ for diaphragm metastasis, $35.2 \%$ and $9.1 \%$ for mesenteric metastasis, and $79.1 \%$ and $65.9 \%$ for retroperitoneal lymph nodes metastasis (Figure $1 \mathrm{~K}$ and $1 \mathrm{~L}$, Table 1 ). As these are the principal sites of metastases of HCC, the superiority of PDOX over free DOX toward metastasis is remarkable and to our knowledge unprecedented, for usually metastases are more resistant than primaries to chemotherapy. It seems likely that the 3:1 molar excess of PDOX over DOX is more visible with metastasis than with the primary tumor because metastatic cells secrete more Cat B per cell than the primary.
In terms of toxicities, PDOX has shown advantages over DOX, even though the dosage of PDOX was 3 times that of DOX in this study. The peripheral blood cells counts such as WBC and PLT levels were significantly higher in the PDOX group than the DOX group, suggesting less bone marrow toxicity of PDOX. Moreover, serum BUN and Cr levels were also significantly lower in the PDOX group than the DOX group, suggesting less renal toxicity of PDOX. The most remarkable observation was the reduced cardio-toxicity in the PDOX group, compared with DOX, as demonstrated by CK, CK-MB, and LDH levels, and histopathological changes. Taking together, these facts support the notion that PDOX has increased anti-metastasis efficacy but reduced toxicities compared with DOX.

In order to explore the potential mechanisms of action of PDOX, we performed an IHC study and western blotting. Among the parameters investigated by $\mathrm{IHC}$, we found that Cat B expression was strong in all tumors, providing supporting evidence that PDOX could produce the effect by this enzyme. Among other parameters related to tumor proliferation and invasion, Ki-67 reduction is the most prominent one in PDOX treated tumors. PDOX could reduce the Ki- 67 positive rate by at least $15 \%$ compared with Control, and by at least $11 \%$ compared with DOX. The Ki-67 is expressed in all the other phases of the cell cycle except G0 phase, making it a reliable marker of active cell proliferation. High expression of Ki-67 has been linked with poor prognosis in prostate, breast, lung and hepatocellular carcinoma [21-24]. Therefore, significant reduction in Ki-67 positive rate could at least account for the fact that PDOX had better tumor inhibition than DOX in this study, although the difference between them did not reach statistical significance.

In addition to tumor proliferation parameters, tumor angiogenesis and lymphoangiogenesis were also studied. The expression of CD34 and VEGF positive endothelial cells may play an important role in understanding the process of angiogenesis in HCC and metastasis [25-27]. D2-40 and E-cadherin may provide important insights into the process of tumor-associated lymphangiogenesis [28-30]. In this study, the expressions of VEGF, CD34, D240 and E-cadherin were positive in all tumors, but there were no statistical differences among 3 groups. Therefore, we speculate that PDOX did not have different effects on tumor angiogenesis, lymphangiogenesis and cell adhesion.

The extracellular signal-regulated kinase (ERK) signaling pathway plays an important role in tumor invasion and metastasis [31,32]. Our study demonstrated that DOX and PDOX reduced ERK phosphorylation and BCL-2, activated casepase- 3 and caspase-9, suggesting that PDOX produced the effect at least via ERK pathway.

Presently, knowledge regarding the biological processes of hepatocarcinogenesis has expanded significantly 
allowing the identification of the molecular processes involved in HCC development. Among these molecules, growth factors and neoangiogenesis factors with their receptors, tyrosine kinase intracellular enzymatic pathways and intracellular signal transmission factors have been under intensive study [33]. These substances represent potential molecular targets for targeted therapies with highly specific small molecules such as sorafenib, sunitinib, brivanib, cetuximab, erlotinib and lapatinib, which have emerged as promising therapeutic approaches for advanced HCC [34,35]. Many other molecular targeting agents to block epidermal growth factor receptor (EGFR), vascular endothelial growth factor receptor (VEGF), platelet-derived growth factor receptor (PDGFR), and mammalian target of rapamycin (mTOR) are also at different stages of clinical development for the treatment of advanced HCC [36-38].

The most successful drug of this kind is sorafenib, an orally-active multikinase inhibitor targeting both tumor cells and the tumor vasculature. It is the first agent to improve the overall survival of patients with advanced HCC, has been approved for molecular targeted therapy for patients with advanced HCC [39], representing a landmark success in the treatment of advanced HCC [40], even though the survival benefit of sorafenib is about 3 months for HCC patients with Child-Pugh Class A liver function, and less infrequent side effects such as hand-foot skin reaction (HFSR) $[41,42]$.

Compared with these small molecules, PDOX could be termed as a "passive targeting agent", which exerts its effect by Cat B cleavage. Normal organs are protected by masking the cytotoxic drug DOX with a simple dipeptide that renders it nontoxic. At the tumor the mask is removed by Cat $\mathrm{B}$, a ubiquitous proteolytic enzyme that is so destructive to tissue that normally it occurs only within cells, encased in lysosomes. Only tumor cells secrete Cat B externally, confined to their plasma membranes, for the purpose of penetrating basement membrane and extracellular barriers during cancer invasion. The prodrug PDOX is rapidly cleaved by Cat $\mathrm{B}$ at the Phe-Lys bond. The resulting PABC-DOX decomposes at once to para-aminobenzyl alcohol, $\mathrm{CO}_{2}$ and free DOX. Furthermore, PDOX kills metastatic cancer cells (which are normally harder to kill than primary tumor cells) more powerfully than free DOX itself.

In summary, this study has provided more supporting evidence to show that PDOX does have increased antimetastatic effects and reduced side effects especially the cardio-toxicity in this highly metastatic HCC model system. PDOX could be a promising new drug candidate for molecular targeting therapy of HCC.

\section{Abbreviations}

HCC: Hepatocellular carcinoma; DOX: Doxorubicin; Cat B: Cathepsin B; PDOX: Ac-Phe-Lys-PABC-DOX; PC: Peritoneal carcinomatosis; FBS: Fetal bovine serum; SPF: Specific pathogen-free; PBS: Phosphate buffered saline; MRI: Magnetic resonance image; ePCl: Experimental peritoneal carcinomatosis index; MVD: Microvessel density; LMVD: Lymphatic microvessel density; ALT: Alanine aminotransferase; AST: Aspartate aminotransferase; GGT: Gamma-glutamyl transpeptidase; TBIL: Total bilirubin levels; DBIL: Direct bilirubin; BUN: Blood urea nitrogen; Cr: Creatinine; CK: Creatine kinase; CK-MB: Creatine kinase-MB; LDH: Lactate dehydrogenase; AFP: Alpha fetoprotein; TACE: Transarterial chemoembolization;

EGFR: Epidermal growth factor receptor; VEGF: Vascular endothelial growth factor receptor; PDGFR: Platelet-derived growth factor receptor; mTOR: Mammalian target of rapamycin; HFSR: Hand-foot skin reaction.

\section{Competing interests}

No potential conflicts of interest were disclosed.

\section{Authors' contributions}

WQ carried out the animal study, the gross pathology and biochemistry study, statistical analysis, and wrote the paper draft. ZYJ carried out the western blotting study and wrote part of the paper draft. YJP carried out the immunohistochemistry study. SLH, ZJ and TL help the animal study and part of the data analysis. LSP help biochemistry study. HYP and FR produced and provided the prodrug. LY designed, supervised and entire study process, and finalized the draft. All authors have read and approved the final manuscript.

\section{Grant support}

This work is supported by The State Key Research Project on Infectious Diseases (2012ZX10002012-012) and the National Natural Science Foundation of China (No. 81171396).

\section{Author details}

'Department of Oncology, Zhongnan Hospital of Wuhan University, Hube Key Laboratory of Tumor Biological Behaviors \& Hubei Cancer Clinical Study Center, No 169 Donghu Road, Wuchang District, 430071 Wuhan, P.R. China. ${ }^{2}$ Princeton Global Synthesis LLC, 360 George Patterson Blvd. Suite 206, 19007 Bristol, PA, USA. ${ }^{3}$ Nanjing Meihua Pharmaceuticals, Ltd, 210009 Nanjing, P.R. China.

Received: 26 June 2013 Accepted: 16 August 2013

Published: 21 August 2013

\section{References}

1. Llovet JM, Bruix J: Molecular targeted therapies in hepatocellular carcinoma. Hepatology 2008, 48:1312-1327.

2. Bruix J, Sherman M: Practice Guidelines Committee, American Association for the Study of Liver Diseases. Management of hepatocellular carcinoma. Hepatology 2005, 42:1208-1236.

3. Yang XR, Xu Y, Yu B, Zhou J, Qiu SJ, Shi GM, Zhang BH, Wu WZ, Shi YH, Wu B, Yang GH, Ji Y, Fan J: High expression levels of putative hepatic stem/prognosis cell biomarker related to tumor angiogenesis and poor prognosis of hepatocellular carcinoma. Gut 2010, 59:953-962.

4. Yeo W, Mok TS, Zee B, Leung TW, Lai PB, Lau WY, Koh J, Mo FK, Yu SC, Chan AT, Hui P, Ma B, Lam KC, Ho WM, Wong HT, Tang A, Johnson PJ: A randomized phase III study of doxorubicin versus cisplatin/interferon alpha- $2 b$ /doxorubicin/fluorouracil (PIAF) combination chemotherapy for unresectable hepatocellular carcinoma. J Natl Cancer Inst 2005, 97:1532-1538.

5. Forner A, Llovet JM, Bruix J: Hepatocellular carcinoma. Lancet 2012 379:1245-1255.

6. Sharma P, Saini SD, Kuhn LB, Rubenstein JH, Pardi DS, Marrero JA, Schoenfeld PS: Knowledge of hepatocellular carcinoma screening guidelines and clinical practices among gastroenterologists. Dig Dis Sci 2011, 56:569-577.

7. Gopinathan A, Denicola GM, Frese KK, Cook N, Karreth FA, Mayerle J, Lerch MM, Reinheckel T, Tuveson DA: Cathepsin B promotes the progression of pancreatic ductal adenocarcinoma in mice. Gut 2012, 61:877-884.

8. Wu D, Wang H, Li Z, Wang L, Zheng F, Jiang J, Zhong H, Huang Y, Suo Z: Cathepsin B may be a potential biomarker in cervical cancer. Histol Histopathol 2012, 27:79-87.

9. Beckham TH, Lu P, Cheng JC, Zhao D, Turner LS, Zhang X, Hoffman S, Armeson KE, Liu A, Marrison T, Hannun YA, Liu X: Acid ceramidasemediated production of sphingosine 1-phosphate promotes prostate 
cancer invasion through upregulation of cathepsin B. Int J Cancer 2012, 131:2034-2043.

10. Lee NP, Chen L, Lin MC, Tsang FH, Yeung C, Poon RT, Peng J, Leng X, Beretta L, Sun S, Day PJ, Luk JM: Proteomic expression signature distinguishes cancerous and nonmalignant tissues in hepatocellular carcinoma. J Proteome Res 2009, 8:1293-1303.

11. Podgorski I, Sloane BF: Cathepsin B and its role(s) in cancer progression. Biochem Soc Symp 2003, 70:263-276.

12. Dubowchik GM, Firestone RA: Cathepsin B-sensitive dipeptide prodrugs. 1. A model study of structural requirements for efficient release of doxorubicin. Bioorg Med Chem Lett 1998, 8:3341-3346.

13. Dubowchik GM, Mosure K, Knipe JO, Firestone RA: Cathepsin B-sensitive dipeptide prodrugs. 2. Models of anticancer drugs paclitaxel (Taxol), mitomycin C and doxorubicin. Bioorg Med Chem Lett 1998, 8:3347-3352.

14. Dubowchik GM, Firestone RA, Padilla L, Willner D, Hofstead SJ, Mosure K, Knipe JO, Lasch SJ, Trail PA: Cathepsin B-labile dipeptide linkers for lysosomal release of doxorubicin from internalizing immunoconjugates: model studies of enzymatic drug release and antigen-specific in vitro anticancer activity. Bioconjug Chem 2002, 13:855-869.

15. Shao LH, Liu SP, Hou JX, Zhang YH, Peng CW, Zhong YJ, Liu X, Liu XL, Hong YP, Firestone RA, Li Y: Cathepsin B cleavable novel prodrug Ac-Phe-Lys$P A B C-A D M$ enhances efficacy at reduced toxicity in treating gastric cancer peritoneal carcinomatosis: an experimental study. Cancer 2012, 118:2986-2996.

16. Li Y, Tang ZY, Ye SL, Liu YK, Chen J, Xue Q, Gao DM, Bao WH: Establishment of cell clones with different metastatic potential from the metastatic hepatocellular carcinoma cell line MHCC97. World J Gastroenterol 2001, 7:630-636.

17. Li Y, Tang Y, Ye L, Liu B, Liu K, Chen J, Xue Q: Establishment of a hepatocellular carcinoma cell line with unique metastatic characteristics through in vivo selection and screening for metastasis-related genes through cDNA microarray. J Cancer Res Clin Oncol 2003, 129:43-51.

18. Monneuse O, Mestrallet JP, Quash G, Gilly FN, Glehen O: Intraperitoneal treatment with dimethylthioampa (DIMATE) combined with surgical debulking is effective for experimental peritoneal carcinomatosis in a rat model. J Gastrointest Surg 2005, 9:769-774.

19. Nerenstone SR, Ihde DC, Friedman MA: Clinical trials in primary hepatocellular carcinoma: current status and future directions. Cancer Treat Rev 1988, 15:1-31.

20. Sciarrino E, Simonetti RG, Le Moli S, Pagliaro L: Adriamycin treatment for hepatocellular carcinoma. Experience with 109 patients. Cancer 1985, $56: 2751-2755$

21. Berney DM, Gopalan A, Kudahetti S, Fisher G, Ambroisine L, Foster CS, Reuter V, Eastham J, Moller H, Kattan MW, Gerald W, Cooper C, Scardino P, Cuzick J: Ki-67 and outcome in clinically localised prostate cancer: analysis of conservatively treated prostate cancer patients from the Trans-Atlantic Prostate Group study. Br J Cancer 2009, 100:888-893.

22. Luporsi E, Andre F, Spyratos F, Martin PM, Jacquemier J, Penault-Llorca F, Tubiana-Mathieu N, Sigal-Zafrani B, Arnould L, Gompel A, Egele C, Poulet B, Clough KB, Crouet H, Fourquet A, Lefranc JP, Mathelin C, Rouyer N, Serin D, Spielmann M, Haugh M, Chenard MP, Brain E, De Cremoux P, Bellocq JP: Ki67: level of evidence and methodological considerations for its role in the clinical management of breast cancer: analytical and critical review. Breast Cancer Res Treat 2012, 132:895-915.

23. Oka S, Uramoto H, Shimokawa H, Iwanami T, Tanaka F: The expression of Ki-67, but not proliferating cell nuclear antigen, predicts poor disease free survival in patients with adenocarcinoma of the lung. Anticancer Res 2011, 31:4277-4282.

24. Stroescu C, Dragnea A, Ivanov B, Pechianu C, Herlea V, Sgarbura O, Popescu A, Popescu I: Expression of p53, Bcl-2, VEGF, Ki67 and PCNA and prognostic significance in hepatocellular carcinoma. J Gastrointestin Liver Dis 2008, 17:411-417.

25. Messerini L, Novelli L, Comin CE: Microvessel density and clinicopathological characteristics in hepatitis $C$ virus and hepatitis $B$ virus related hepatocellular carcinoma. J Clin Pathol 2004, 57:867-871.

26. Amarapurkar AD, Vibha V, Kim V: Angiogenesis in liver cirrhosis and hepatocellular carcinoma. Indian J Pathol Microbiol 2008, 51:323-328.

27. Zhong C, Guo RP, Shi M, Wei W, Yu WS, Li JQ: Expression and clinical significance of VEGF and MMP-9 in hepatocellular carcinoma. Ai Zheng 2006, 25:599-603.
28. Tanaka N, Odajima T, Ogi K, Ikeda T, Satoh M: Expression of E-cadherin, alpha-catenin, and beta-catenin in the process of lymph node metastasis in oral squamous cell carcinoma. Br J Cancer 2003, 89:557-563.

29. Tretiakova MS, Hart J, Shabani-Rad MT, Zhang J, Gao ZH: Distinction of hepatocellular adenoma from hepatocellular carcinoma with and without cirrhosis using E-cadherin and matrix metalloproteinase immunohistochemistry. Mod Pathol 2009, 22:1113-1120.

30. Dumoff KL, Chu C, Xu X, Pasha T, Zhang PJ, Acs G: Low D2-40 immunoreactivity correlates with lymphatic invasion and nodal metastasis in early-stage squamous cell carcinoma of the uterine cervix. Mod Pathol 2005, 18:97-104.

31. Ito $Y$, Sasaki $Y$, Horimoto M, Wada S, Tanaka Y, Kasahara A, Ueki T, Hirano T, Yamamoto H, Fujimoto J, Okamoto E, Hayashi N, Hori M: Activation of mitogen-activated protein kinases/extracellular signal-regulated kinases in human hepatocellular carcinoma. Hepatology 1998, 27:951-958.

32. Reddy KB, Nabha SM, Atanaskova N: Role of MAP kinase in tumor progression and invasion. Cancer and Metast Rev 2003, 22:395-403.

33. Rossi L, Zoratto F, Papa A, lodice F, Minozzi M, Frati L, Tomao S: Current approach in the treatment of hepatocellular carcinoma. World $J$ Gastrointest Oncol 2010, 2:348-359.

34. Zhu AX: Systemic treatment of hepatocellular carcinoma: dawn of a new era? Ann Surg Oncol 2010, 17:1247-1256.

35. Tanaka S, Arii S: Current status of molecularly targeted therapy for hepatocellular carcinoma: basic science. Int J Clin Oncol 2010, 15:235-241.

36. Zhu AX: Beyond sorafenib: novel targeted therapies for advanced hepatocellular carcinoma. Expert Opin Investing Drugs 2010, 19:663-672.

37. Rimassa $L$, Santoro A: The present and the future landscape of treatment of advanced hepatocellular carcinoma. Dig Liver Dis 2010, 42:273-280.

38. Wörns MA, Schuchmann M, Düber C, Otto G, Galle PR, Weinmann A: Sunitinib in patients with advanced hepatocellular carcionma after progression under sorafenib treatment. Oncology 2010, 79:85-92.

39. Wilhelm SM, Carter C, Tang L, Wilkie D, McNabola A, Rong H, Chen C, Zhang X, Vincent P, McHugh M, Cao Y, Shujath J, Gawlak S, Eveleigh D, Rowley B, Liu L, Adnane LM, Lynch MD Auclair D, Taylor I, Gedrich R, Voznesensky A, Riedl B, Post LE, Bollag G, Trail PA: BAY 43-9006 exhibits broad spectrum oral antitumor activity and targets the RAF/MEK/ERK pathway and receptor tyrosine kinases involved in tumor progression and angiogenesis. Cancer Res 2004, 64:7099-7109.

40. Cheng AL, Kang YK, Chen Z, Tsao CJ, Qin S, Kim JS, Luo R, Feng J, Ye S, Yang TS, Xu J, Sun Y, Liang H, Liu J, Wang J, Tak WY, Pan H, Burock K, Zou J, Voliotis D, Guan Z: Efficacy and safety of sorafenib in patients in the Asia-Pacific region with advanced hepatocellular carcinoma: a phase III randomised, double-blind, placebo-controlled trial. Lancet Oncol 2009, 10:25-34.

41. Fukumoto T, Ku Y: Induction Chemotherapy for Hepatocellular Carcinoma. Berlin: Springer-Verlag; 2011:237-250.

42. Lacouture ME, Wu S, Robert C, Atkins MB, Kong HH, Guitart J, Garbe C, Hauschild A, Puzanov I, Alexandrescu DT, Anderson RT, Wood L, Dutcher JP: Evolving strategies for the management of hand-foot skin reaction associated with the multitargeted kinase inhibitors sorafenib and sunitinib. Oncologist 2008, 13:1001-1011.

doi:10.1186/1479-5876-11-192

Cite this article as: Wang et al:: Targeting therapy of hepatocellular carcinoma with doxorubicin prodrug PDOX increases anti-metastatic effect and reduces toxicity: a preclinical study. Journal of Translational Medicine 2013 11:192 